\title{
Advancing Design Science Research with Solution-based Probing
}

\author{
Robert O. Briggs* \\ San Diego State Univ. \\ MIS Department \\ rbriggs@sdsu.edu
}

\author{
Tilo Böhmann* \\ University of Hamburg \\ Department of Informatics \\ tilo.boehmann@uni-hamburg.de
}

\author{
Gerhard Schwabe* \\ University of Zurich \\ Dep. of Informatics \\ schwabe@ifi.uzh.ch
}

\author{
Tuure Tuunanen* \\ University of Jyväskylä \\ Faculty of IT \\ tuure@tuunanen.fi
}

\begin{abstract}
We propose solution-based probing as an extension of action design research. The core idea is that researchers bring a prototype solution (probe) into one or more fields and explore to synthesize robust and generalizable design knowledge, along with knowledge of the phenomena and correlations we discover. We believe proposing solutions creates opportunities for researchers to innovate and to document the impact. In addition, solutions can be effective probes for advancing theory, in terms of design theories and in creating exploratory foundations for behavioral and causal theory. We illustrate solution-based probing with four exemplar studies in the areas governance of municipalities, police work in informing citizens, learning in public schools, and naval decision making. We identify critical activities for ideating and initiating solutionbased probing and for deriving sustainable solutions and scholarly knowledge from such studies. Finally, we discuss future directions for improving researchers' ability to conduct high-impact solutionbased probing research.
\end{abstract}

\section{Introduction}

Rigor and relevance need not be mutually exclusive in information systems (IS) research. Design science research (DSR) scholars have an ongoing quest to increase the relevance and impact of IS research. Toward that end, DSR researchers have produced a substantial stream of methodological advances in recent years. However, DSR has not yet produced levels of documented methodological knowledge comparable to that for other research paradigms. When DSR ventures beyond the lab to create socio-technical design knowledge, the research process can grow complex, because it requires that researchers pursue two simultaneous goals that some believe are mutually exclusive: rigorous academic contribution and valuable societal or business impact.

We propose a particular approach to design science research: solution-based probing. In this, researchers take a prototype solution (probe) into one or more fields where an important class of unsolved, wicked problems persists, and conduct rigorous exploratory research to learn what happens. They observe phenomena that define the problem and explore the solution space. Researchers use abductive and deductive logic to inform counter-intuitive design choices. The DSR researcher tests theory-informed solutions with rigor, contributing generalizable design knowledge, and scholarly knowledge of phenomena. Early solution-based probing studies of wicked problems are likely to be technological failures and scholarly bonanzas, as researchers discover the complexity of the problem space. Later studies are likely to bring more technological success and deeper scholarly understandings of the phenomena.

We use the term solution to denote a sociotechnical artifact whose primary purpose is to improve outcomes for some problem. Thus, the focus of solution-based probing is shaping socio-technical innovations in field settings for the value the innovations can create for end users and for society ("proof of value") and their operational feasibility ("proof of use") [1]. Such an approach can satisfy the dual goals of DSR: advancing theory and evolving sustainable socio-technical innovations that are used beyond the research context. This approach instantiates action design research (ADR) [2] while leveraging insights from the DSR method (DSRM) [3]. Solution-based probing can begin when a working prototype can be taken into the field. A solution probe may come from any source, be it previous ADR cycles or inspired intuition. Solution-based probing is consistent with an objectives-based DSRM, one of the four styles of DSR defined by Peffers et al. [3].

${ }^{*}$ Authors in alphabetical sequence. All authors have contributed equally to this paper. 


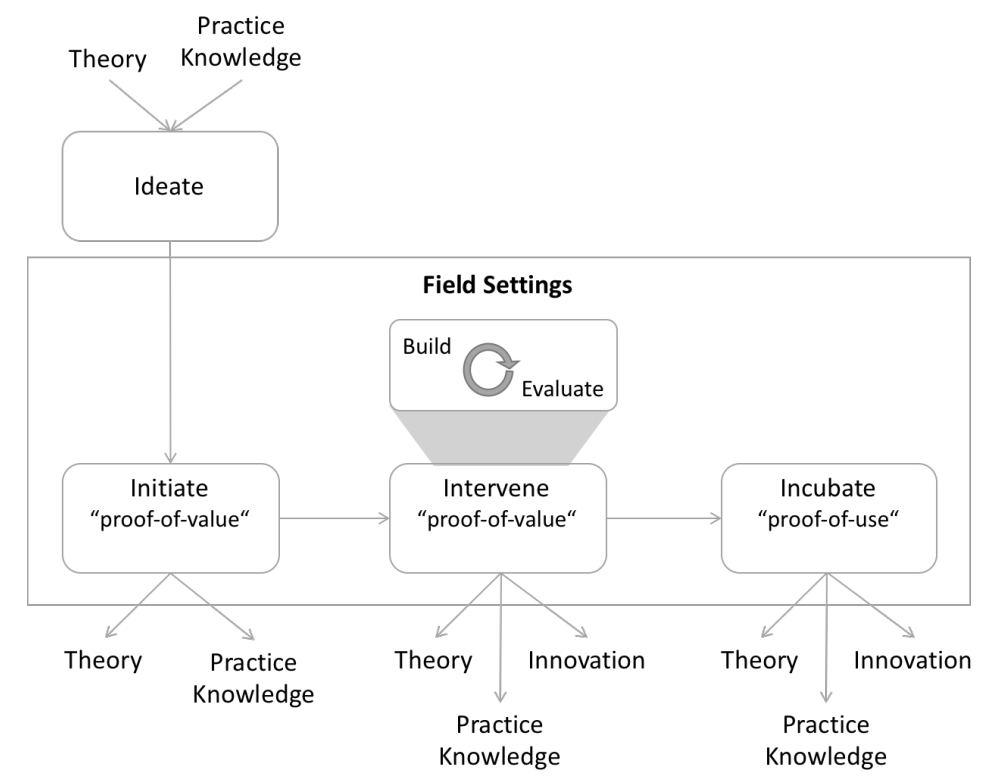

Figure 1. The solution-based probing design science research process

\begin{tabular}{|c|c|c|c|c|}
\hline & Cuparla & SmartProtector & Cognito & USS Coronado \\
\hline \multirow{3}{*}{ 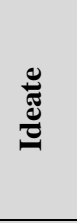 } & $\begin{array}{l}\text { Objective: Demonstrate } \\
\text { potential of telework }\end{array}$ & $\begin{array}{l}\text { Objective: Support } \\
\text { police officers' work }\end{array}$ & $\begin{array}{l}\text { Objective: Reduce the dropout } \\
\text { rate in schools }\end{array}$ & $\begin{array}{l}\text { Objective: Shorten } \\
\text { decision cycles }\end{array}$ \\
\hline & Artifact: Application & Artifact: Application & Artifact: Group support systems & $\begin{array}{l}\text { Artifact: Collaborative } \\
\text { decision technologies }\end{array}$ \\
\hline & $\begin{array}{l}\text { Solution: Modify working } \\
\text { structures }\end{array}$ & $\begin{array}{l}\text { Solution: Modify police } \\
\text { officers' work }\end{array}$ & $\begin{array}{l}\text { Solution: Change motivation to } \\
\text { learn }\end{array}$ & $\begin{array}{l}\text { Solution: Change concepts } \\
\text { of operations }\end{array}$ \\
\hline 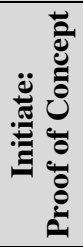 & $\begin{array}{l}\text { Prototype system with } \\
\text { new IBM notebooks, a } \\
\text { connection to the city } \\
\text { administration via ISDN, } \\
\text { and access to the internet }\end{array}$ & $\begin{array}{l}\text { SmartProtector app } \\
\text { with functionalities in } \\
\text { an easy-to-understand } \\
\text { user interface. }\end{array}$ & $\begin{array}{l}\text { Previous research showed that by } \\
\text { using group support systems } \\
\text { teams could gain } 90 \% \text { savings on } \\
\text { project cycle times and reduce } \\
\text { labor hours by } 50 \% \text { while } \\
\text { producing superior work output. }\end{array}$ & $\begin{array}{l}\text { A workshop planned for } \\
\text { two days was completed } \\
\text { with a thorough plan } \\
\text { within two hours. }\end{array}$ \\
\hline 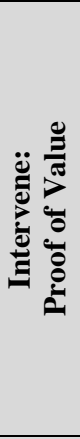 & $\begin{array}{l}\text { Continuous collaboration } \\
\text { with the city councils led } \\
\text { to increasingly more } \\
\text { mature versions of the } \\
\text { applications and deep } \\
\text { trust and openness } \\
\text { between the council } \\
\text { members and parts of the } \\
\text { administration. }\end{array}$ & $\begin{array}{l}\text { Tablet application was } \\
\text { provided to active-duty } \\
\text { police officers to offer } \\
\text { them additional ways to } \\
\text { demonstrate to citizens } \\
\text { how easy it is to break } \\
\text { into an unprotected } \\
\text { house and how } \\
\text { protection measures } \\
\text { help. The application } \\
\text { was extended to other } \\
\text { areas of police work. }\end{array}$ & $\begin{array}{l}\text { Based on the credibility we } \\
\text { gained from the trials, we } \\
\text { received a grant to support a two- } \\
\text { year demonstration project in the } \\
\text { school and acquired sufficient } \\
\text { funding to put computers in } \\
\text { every classroom in the school } \\
\text { and to outfit two special } \\
\text { classrooms as collaborative } \\
\text { learning spaces. }\end{array}$ & $\begin{array}{l}\text { New quiet collaborative } \\
\text { decision spaces aboard the } \\
\text { ship each optimized for } \\
\text { different planning } \\
\text { horizons and concepts of } \\
\text { operation to reduce } \\
\text { decision cycles. } \\
\text { However, staff declined to } \\
\text { use the technology on } \\
\text { their own because it was } \\
\text { too difficult to use without } \\
\text { help. }\end{array}$ \\
\hline 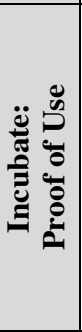 & $\begin{array}{l}\text { The system became an } \\
\text { indispensable part of the } \\
\text { Stuttgart city council's } \\
\text { work practice relevant due } \\
\text { to the } 20 \text { years of use. } \\
\text { However, the pilot test } \\
\text { was never completed, and } \\
\text { commercialization failed. }\end{array}$ & $\begin{array}{l}\text { A larger research } \\
\text { stream on advisory } \\
\text { support concluded } \\
\text { proof-of-use validation, } \\
\text { and the } \\
\text { commercialization } \\
\text { project successfully } \\
\text { transferred knowledge } \\
\text { to a software company. }\end{array}$ & $\begin{array}{l}\text { At the end of the project, the } \\
\text { students were two years ahead of } \\
\text { their peers in reading and writing } \\
\text { skills, and the students were } \\
\text { highly advanced in their } \\
\text { collaborative problem-solving } \\
\text { skills. All participants in the } \\
\text { project stayed in school. }\end{array}$ & $\begin{array}{l}\text { Many concepts from the } \\
\text { solution-based probing } \\
\text { studies were integrated in } \\
\text { a new class of Navy } \\
\text { command ships. }\end{array}$ \\
\hline
\end{tabular}

Table 1. Summary of Four Solution-based Probing Exemplar Studies 
In this paper, we draw on decades of experience with solution-based probing in the field to offer howto knowledge instantiating higher-level DSR frameworks such as ADR and the DSRM. We present the details of four exemplar studies and provide a comparative analysis of key challenges in different phases of solution-based probing. We illustrate the utility of solution-based probing to create opportunities to innovate and to create documented impact. We show how this can facilitate access to field settings. Thus, practitioners can partner with academia in ways that are compatible with organizational innovation processes, which, in turn, leads to additional novel methods of creating value for practitioners. We describe instances where solutionbased probing advanced design knowledge and scholarly knowledge, and in some cases, inspired new behavioral theory.

Previous works have examined how to frame a pilot study $[1,4]$ and how to embed a pilot study in a design science project [5]. There is a gap in the extant literature, however, on how to conduct solution-based probing in service of DSR and how such studies lead to generalizable DSR knowledge.

\section{Solution-based probing}

A core tenet of solution-based probing is to invent a novel socio-technical solution and use it in one or more pilot studies to derive sociotechnical construction principles and scholarly knowledge before making the solution available to a larger user community. Solutions can serve as a probe for assessing the viability of an innovation, foster a deeper understanding of socio-technical design principles, and facilitate theory development based on sociotechnical insights from field settings. Moreover, solution-based probing is not bound to a single organizational context but seeks to contribute to generalizable design knowledge by piloting in different organizational settings or in organizational ecosystems. Solution-based probing builds on the tradition of piloting research that advocates comprehensive pilot research studies to build sociotechnical design knowledge and understand the effects of innovations before rolling them out to the intended users at large $[4,6]$.

Solution-based probing comprises four critical phases (see Figure 1), which start with ideation, or invention. Based on theoretical logic, factual knowledge, and creative intuition, researchers conceive a design for a generalizable solution. The design may embed theoretical knowledge (e.g., theory-driven design) and the researchers' unique ideas about novel ways of approaching a particular problem or challenge. The idea can be novel technologically and/or socially. The initiation phase produces early expository instances of the generalizable solution. The primary goal is proof of concept, to find out whether the solution is technically feasible. Pilot studies of the solution in field settings look for phenomena related to effectiveness and efficiency, and explore for issues that could impact acceptance and utility.

Central to solution-based probing are pilot studies in at least one and ideally multiple field settings. Initiating these pilot studies is an important part of the overall research process. The initiation phase involves persuading multiple stakeholders, such as the managers and staff in organizations, to participate in the pilot study. Moreover, initiation is about starting to engage these stakeholders in active exploration of the solution and the evolution of the design of the solution.

The intervention phase takes a robust prototype into the field seeking proof of value-which practitioners can use the solution to create real value, albeit with the support of the research team. Researchers explore and further develop the solution based on formative evaluation. This phase is a typically cyclical progress of building, intervening, and evaluating [2]. The continual process of reflection and learning deepens the researcher's knowledge of the problem the solution seeks to address of the design of the socio-technical solution itself.

The intervention phase transitions into the incubation phase. Researchers compile generalizable scholarly knowledge from the pilot studies and test the new insights in the field. Thus, a rich body of scholarly knowledge emerges. The primary focus is gaining proof of use: to show that a solution has matured sufficiently to attract a growing and self-sustaining community of practice apart from the researchers who invented the solution. Success often depends not on discovering one big thing to do right but on doing many small things right. The incubation phase seeks to discover, codify, and address the many small things that must be done well for the solution to create value. In this phase, researchers often discover that without noticing, they have hardcoded a number of unnoticed and counter-productive assumptions into previous versions of the solution. Thus, new generations of technology and practice emerge. Proof of value enables researchers to persuade field partners to commercialize viable solutions.

Although the incubation phase clearly focuses on generalizable knowledge and new solutions, we have observed that solution-based probing can generate novel insights for theory and practice in previous phases. The interaction between a socio-technical 
artifact and field settings is a fertile ground for generating theory, innovating, and uncovering practice knowledge.

\section{Solution-based probing in action}

Publications on solution-based probing are rare. Therefore, in this section, we present four solutionbased probing research programs that we have conducted over the past 30 years. They were particularly interesting cases led by two of the authors. The cases fulfill the following purposes: 1) The cases depict what we mean by solution-based probing, 2) they serve as an empirical basis for the process of development of the core concepts of solution-based probing, and 3) the four cases distinguish between the recurrent patterns and idiosyncratic features of each case. The first case, Cuparla, describes how solutionbased piloting was used to modify work structures in German municipalities. The second, SmartProtector, yielded improvements in police work in a Swiss canton. The third case, Cognito, used collaboration technologies and methods to reduce dropout rates among students in the United States. The fourth case, the USS Coronado project, enhanced decision making aboard a U.S. Navy command ship. Table 1 summarizes the details.

\subsection{Cuparla telework for German municipalities}

In 1995, German Telekom launched a set of projects to explore the potential of "telework," that is, working from home. One of those projects was Cuparla, which aimed to support the work of city councilors [7]. One of the authors led the program. The IDEATION phase began with deliberations by the city council of Stuttgart, Germany, who then agreed host a pilot site. Kornwestheim, a nearby city, agreed to set up a second pilot site. Having two pilot sites not only served as a safeguard against the potential failure of one site but also allowed the researcher to distinguish between idiosyncratic properties of one site vs. recurring patterns of behavior across sites. Furthermore, two pilot sites created a spirit of healthy competition between the cities and the factions within the cities [8].

The INITIATE phase of solution-based probing started with the proof-of-concept system that turned out to be promising. Very early on, a subset of the council members were equipped with new fancy IBM notebooks, a connection to the city administration via ISDN, and access to the (at that time very new) Internet. We were surprised how tolerant the council members were of technical difficulties with the pilot system if they were faced with the option of returning the notebook if they deemed the trouble unbearable. Having a notebook and a connection to the Internet had become a part of their personality. These pilots revealed many challenging issues that had to be addressed, among them managing information asymmetry and organizing collaboration.

The INVERNE phase of solution-based probing focused on these issues. The team considered a workflow solution but ultimately rejected the idea because the challenges were too complex and ambiguous to formalize, and the solution would be too complex and non-transparent. The team discovered that previous work practices were organized around physical locations, such as a home office, faction room, etc. The first pilot application mimicked this structure, letting teleworkers move documents from one "location" to the next. The application organized information access with physical metaphors. For example, information in your "faction room" feature could be seen only by your faction.

Working closely with the city council, the researchers developed increasingly mature iterations. This built deep trust and openness between the council members and other parts of the administration, which, in turn, made collecting data for research easy.

From one perspective, the INCUBATE phase produced proof-of-use success for Cuparla. It became indispensable to the Stuttgart city council's work practices and has been in continuous use for more than 20 years. From another perspective, the project was a failure. It fell into the "pilot test never ends" trap. The pilot test never transitioned to any other groups or organizations. The city of Stutgart never saw the necessity to transition the solution to other entities. Instead, the university and a spin-off company continued to support Cuparla for years. A subsequent commercialization project failed to grasp the nuances of the design and use. No other city rolled the project as out as proof of use. Therefore, the research is not yet complete.

\subsection{SmartProtector}

In many European countries, safety-aware home owners can call a special local police officer to give them advice on how to make their homes more burglary-proof. In 2012, one of the authors launched a project to support the police officers' work by providing them with a tablet computer and advisorysupporting software [9]. The initial solution was informed by a previous research stream on advisory support systems. 
As part of the IDEATE phase, the researchers conducted a study of the traditional work practices and quickly developed a proof-of-concept prototype.

The INITIATE phase started with the first SmartProtector prototype. Following the principle of a minimal viable prototype, this tablet application offered police officers functionalities a) to document security issues by taking pictures and drawing on them, b) to select appropriate solutions (from a database) and explain them to home owners, c) to collaboratively plan the implementation of the security measures, and d) to send a PDF document with all documentation to the home owners. All functionality was embedded in a simple-to-understand interface.

During the INTERVENE phase, the initial field tests with two police officers (and 12 volunteer home owners) showed promising results and uncovered an interesting effect: Taking and sharing pictures could reduce location-dependent forgetting. In the next step, we had one advisor star in a video showing that a burglar could break into a house with just a screwdriver in 60 seconds. The video had two effects: It motivated homeowners to participate in the advisory experience, and it put the advisor in the spotlight. This motivated the advisor to start using the tablet in his daily work as soon as we could provide him with a stable version.

It took some time to discover the most important value of the video. The dirty secret of police home security advice is that all home owners love it, but few implement the recommended security measures. "Advice discounting" was a big problem. Once we understood this, we reimagined the system as a new class of persuasive technology and added several features for that purpose. The biggest persuaders turned out to be videos showing break-ins and how protection measures helped. We added four videos to the next release and promoted them to the other users.

Soon after the first pilot began, advisors from the Canton of Zurich, two other Swiss cantons, and two German states joined the project. Researchers capitalized on this increase in interest to gather publishable data. They invited police officers from each new site to a two-day training session. On the first day, the officers were trained to adapt their work practices to the new tool. On the second day, the officers conducted six to eight experimental sessions, three to four applications of the tool and three to four conventional control sessions, with test clients. This training was meaningful for the officers. For the researchers, the training was an opportunity to gather controlled test data for a proof-of-value evaluation. Training the professional police advisors turned out to be a challenge. A third of the police officers did not return after the first training day. The officers did not think that "those academics can tell us how we work." Thereafter, the researchers integrated an experienced police advisor into the training team. Credibility has not been an issue since.

By 2015, it had become clear to all stakeholders that the SmartProtector was and should be there to stay. The researchers tried to avoid the "pilot test never ends" pitfall by taking two measures during the INCUBATE phase: charging a high amount of money for maintaining the systems after the end of the official pilot project and explaining to the users and their IT support staff why continued use of the pilot software would be unnecessarily expensive. These measures persuaded the stakeholders to start a commercialization project. Some researchers accompanied the commercialization project "on the way out" and thus, assured knowledge transfer to a software company. At the time of this writing, the commercial software has been rolled out for a year, and the pilot test has officially been terminated. The commercial software is used in the field daily in multiple countries. Thus, the solution-based probe program gained proof-of-use validation.

\subsection{Cognito: Reducing the dropout rate}

In 1992, Washington D.C. Public Schools (DCPS) had a $64 \%$ dropout rate. More than half of the children who started school in the school system never finished in any district. Previous research suggested that the students who dropped out did not believe in the future for which the school tried to prepare them. One student said:

I got on the wrong side of a gang today, so I don't know if I'm going to get home alive tonight. If I get home alive, I don't know if my mom will be strung out on heroin. If she's strung out on heroin, I don't know whether I'll get anything to eat before I get back to school Monday morning. What do I care what $7 \times 5$ is? What do I care when Columbus sailed? This has nothing to do with me!"

This was a common situation in large U.S. cities. Children who stay in school are more likely to find employment, break out of poverty, contribute to the prosperity of the community, and educate their own children. Education could be the road out of poverty for a community, but many poor children reject education. A solution might yield substantial benefits. We ran a multi-year project as a series of small studies, each aimed at the larger goal.

For the INITIATE phase, one of the authors joined veteran DCPS Administrator Howard M. Brown in running a solution probing project to find a way to reengage the learners at risk. He was a respected classroom veteran and administrator with a passion for 
solving the problem. He knew the power players in the DCPS and the local and federal governments, knew the history of previous failed initiatives, and participated actively in all aspects of the research.

Drawing on a nascent theory of learning that proposed motivation to learn as a function of perceived vested interest in the content, we reasoned that if students did not believe in the future for which they were being prepared, we should stop preparing them for that future and put them to work on real problems in which they perceived an immediate vested interest. We should choose problems such that the students would need to learn what we wanted them to know to attain their goals. However, there was not enough time in a conventional classroom to work on real problems. Teachers had time only to deliver content and test whether students remembered it. Therefore, the teachers gave students small, hypothetical problems that did not interest them. Research showed, however, that new collaboration systems could shorten business decision cycles by $90 \%$. We reasoned that this same technology might give students just enough of an edge to solve small but real problems in the classroom. An unfunded proof-ofconcept field trial yielded promising results. Administrator Brown persuaded the school system and government agencies to grant us access and resources to pilot the approach in some classrooms.

In the INTERVENE phase, we conducted proofof-value collaborative learning experiences in junior high and high schools. Students were enthusiastic about using the system to solve real problems, and they learned the intended content. Many students dropped out, however, before they reached high school. Based on evidence gained from the trials, we received a grant for a two-year demonstration project in Orr Elementary School in the Anacostia neighborhood. The grant gave funding for computers in every classroom (rare in those days) and to outfit two special classrooms as technology-supported collaborative learning spaces. One fifth grade and one sixth grade were selected to participate in the project.

To learn the students' current vested interests, we ran online brainstorming sessions on three consecutive days, asking questions such as the following:

"What do you want to be when you grow up and why?"

"Who is your favorite hero from history, and why?"

"Who is your favorite living hero, and why?"

"What's the funniest thing that ever happened to you?"

Their answers helped us choose learning problems that would motivate them. We used the information to show the students how to get something they wanted by working on a project. In the preliminary brainstorming sessions, for example, we had discovered, among other things, that the students were eager to achieve greatness. Each wanted to become a great athlete, a great cop, a great hairdresser, a great teacher. On the first day of formal learning, we told the students, "This year, we won't study anything boring, or irrelevant, or unimportant. If we get into something like that by accident, just let us know. We will stop and work on something more important." A child responded, "History! History is boring! We won't have to study history, right?" We had anticipated this response and had something up our collective sleeve. We answered: "If history isn't important, we won't study it. But people have been studying it for more than 800 years. Let's explore whether history is worth studying and then decide." The students went online for an anonymous brainstorm on the question, "Is history worth studying?" Some were skeptical: "What could we learn from men who wore white wigs and silk stockings?" Others were tepid: "Well, I guess you need to know about your roots."

At the end of an hour, the students ran out of ideas. We proposed a real problem that tapped into a common vested interest: "History," we said, "is a shortcut for achieving greatness. Each of you plans to achieve greatness in something. Why don't we dig into the lives of your favorite heroes from history to find out how they achieved greatness? We can use this collaboration system to write a book together. We could call it, Achieving Greatness in Anacostia. You can explain how your heroes from history achieved greatness, and then, at the end, you can each write a chapter on the topic, "What must I do to achieve greatness in Anacostia?'”

The students were lukewarm about the idea. We were not yet done. Their task had to be a real problem. Thus, we said, "We will put the book in the school library when you finish it, so other children your age can learn from it." They were slightly more interested but not ready to commit. We said, "And why don't we send copies of the book to all your living heroes to see if they respond and perhaps even visit our school." One asked, "Can we start right now?" And we did.

The students generated a list of their heroes from history and held an electronic vote to decide which hero to work on first. They chose Dr. Martin Luther King Jr. We set up a collaborative speed-poetry competition to create the first two contributions to the book. First, they brainstormed and then narrowed their list to 13 attributes that made Dr. King great. This became the frontispiece for the book. Then the students paired up at computers. Each team got one of the attributes as a starting point. We agreed on a meter for the poem. On a signal, they raced to see which team 
could compose and submit a stanza first. The poem automatically assembled on a public screen as each team contributed. It was dreadful. The students laughed hard when they read it aloud in unison. Two students volunteered to clean up the scan, the meter, and the rhymes. At the end of the day, we hung a framed copy on the wall with all their signatures. We declared them poets and added the poem to their book. It was a task in which they had an immediate vested interest.

When they finished the book several months later, they sent copies to their living heroes. They got a personal letter back from President Bill Clinton and a visit from General Don Lynch, then second-incommand of the U.S. Marine Corps. He brought with him Colonel Charles Bolden, USMC, the first black astronaut and shuttle pilot (and later the director of NASA). We structured the subsequent learning activities in a similar way.

At the end of two years, the collaborative problemsolving students were two years ahead of their peers in reading and writing skills, were comparable on standardized tests of declarative knowledge (such as social studies and history), and had gained advanced collaborative problem-solving skills their peers in comparable classrooms lacked. We followed their progress for five years. All project participants graduated high school, while more than half of their peers dropped out.

This success gave us proof of value for the solution, but curiously, the INCUBATE phase was a failure. We were not able to persuade a single teacher to attempt a similar approach, even after a month of fully paid summer training. None of the easy explanations for the teachers' reluctance turned out to be sound. It remained a mystery for some years. We discovered an answer while working with the U.S. Navy and the USS Coronado.

\subsection{USS Coronado}

One of the authors led a field investigation in an effort to integrate collaborative decision technologies and concepts of operations into the daily work of the U.S. Navy's Third Fleet (COMTHIRDFLT) aboard the USS Coronado, the command ship for COMTHIRDFLT [10]. The Coronado's mission was to take every action possible to forestall the outbreak of a shooting war. The ship served as a floating command center not only for COMTHIRDFLT staff but also for temporarily embarked flag and general staffs, diplomats, and non-government organizations. When a crisis broke out, the ship would move to the center of crisis and invite representatives of all stakeholders aboard to negotiate a resolution. Halfway through the project, another researcher, Mark Adkins, took on the leadership role through the completion of the project.

Command staffs and other crisis responders face problems of such complexity that no single officer or government official can accomplish them alone. This work places the officials under a high cognitive load and severe time pressure. Previous research with industry, non-profit, and academic institutions showed that collaborative decision technologies could enable discontinuous improvements in the time on task and the quality of the deliverables. To IDEATE a solutionbased probing study, we proposed to explore the use of collaborative decision technologies to support military and diplomatic decision-making processes under time pressures. The project was interesting to military personnel for an additional reason. It is an axiomatic doctrine that the military that can think and act the fastest can prevail against even a physically superior adversary by forcing their adversary to respond to conditions that no longer exist. We predicted we might be able to shorten the decision cycles aboard the ship by half or two-thirds with judicious applications of work practices supported by collaborative decision technologies.

The INITIATE phase of solution-based probing began with a meeting with Capt. Richard Williams (COMTHIRDFLT, USN). During our first meeting, we asked whether he might be planning to take a team off the ship for, perhaps, a two-day workshop, a common practice for ships in port. He said they had planned a two-day off-site event the following week to make preliminary plans for moving the ship to a new home port, a massive logistical challenge. We offered to support the workshop in a group support system lab and suggested they might be able to finish the project in one morning. They planned for two days anyway. They were finished with a thorough plan within 2 hours. Capt. Williams invited us to come aboard the ship to work on additional projects. Our research team integrated with the fleet staff for the next six years.

In the INTERVENE phase of solution-based probing, we surveyed the fleet staff and the ship's company to discover their most aggravating problems. We discovered several major issues: 1) The ventilation systems in the existing command spaces peaked as high as 110 decibels, approximately the same volume as the flight deck of an aircraft carrier. In addition to not being able to hear one another to collaborate, the staff members were sustaining permanent hearing loss. 2) Computers were all secured in stands welded to the bulkheads, so people could not see their computers and one another simultaneously. 3) Fleet personnel were scattered in tiny offices throughout the ship and tended to communicate only with people in their own office. 
Thus, the left hand did not know what the right hand was doing. Antisubmarine forces, for example, tended to sink their own submarines in training exercises.

Next, we designed and built four new quiet collaborative decision spaces aboard the ship each optimized for different planning horizons: one month to one year, one week to one month, one day to one week, and moment to moment. We developed new collaboration technologies and new concepts of operation to reduce the decision cycles. For example, working with the intelligence unit, we developed a process that cut the situational awareness cycle from 30 minutes, which, at the time, was regarded as world class, to 1 minute. Working with the battle staff, we designed a collaborative course-of-action development approach that cut the cycle from 90 minutes to 30 minutes, while quadrupling the number of viable options the staff could develop.

The intelligence group adopted the collaboration technology after only 1 hour of training, and the new approach spread quickly across the fleet. The planning staff worked with the research team 18 hours a day during deployments that lasted 2 to 6 weeks, over a period of several years, and derived a great deal of value from the technology. However, similar to the teachers in the Anacostia neighborhood, the staff declined to use the technology on their own. When we asked them why, they said that it was too difficult to use without help.

This curious difference between the intelligence and planning groups became the key that unlocked the mystery. Toward the end of the third year of the project, a Navy commander opined, "You would have to have a Ph.D. to run this technology." We replied, "I don't understand. The intelligence folks have been using it 24/7 for more than a year with only 1 hour of training. Fifth- and sixth-graders in D.C. public schools ran it for themselves after two or three days of watching us do it. What makes it seem so hard to you?" The commander explained, "Look, I can't start up this technology in a room full of captains and admirals, when I don't know what's going to happen. I see what you do with the technology every day, and I see that it works, but I don't know why you do and say those things, and I don't know why they work. It could cost me my career if things went badly."

It was a moment of revelation. The tools themselves were easy enough to operate. The effective, efficient collaborative work practices, however, were not self-evident. For the intelligence community, we had, without realizing it, designed a repeatable process for a high-value recurring task. It used the simplest feature of the system. It took only an hour to learn that process, and they did not need to know anything else about the system to succeed. The planning group, in contrast, addressed multiple idiosyncratic problems a day, each of which required a different work practice using a different configuration of the system. The system, we calculated, had more than 10 million possible configurations. Later field work revealed that about 1 in 20 people have an intuitive grasp of collaboration. To them, it seemed obvious what to do with the tools. It was not clear to the other 95 percent of the population what one could do with the tools.

This brief conversation with the Navy commander sparked the INCUBATE phase of solution-based probing and a research stream now called Collaboration Engineering, an approach to designing technology-supported work practices for high-value recurring tasks and transferring them to practitioners to execute for themselves without ongoing support from a collaboration expert. Our understanding of the challenges faced by fleet personnel evolved with experience. Therefore, we adapted the problem statements and designed the goals to reflect the changes. At the end of the project, many concepts from the solution-based probing studies were integrated into a new class of Navy command ships, establishing proof of use.

\section{Discussion}

Solution-based probing yields valuable theoretical knowledge in three ways. First, this probing aids the generation of more comprehensive design theory for a particular domain. Second, solution-based probing provides the opportunity to stipulate relevant generalizable design theory based on broad interaction with users and stakeholders in field settings. Third, solution-based probing can stimulate the development of behavioral theory from in-depth field experiments on human use of technology.

This study contributes to the DSR literature by showcasing how ADR [2] concepts can be leveraged in field studies that do not necessarily allow a researcher to be embedded with the organization while the organizations or their customers are using the artifact. We extend the current conceptualization of ADR by proposing research phases that happen before and after the currently recognized stages of problem formulation, intervention (build-evaluation), reflection and learning, and formalization of learning.

We argue that to conduct solution-based probing researchers need to emphasize the INITIATE phase. The four exemplar studies depict various problems that we have faced and how we resolved them. Across all the studies, we can see that there is a requirement to foster relationships with the current and potential 
research clients at the institutional level. This issue is often overlooked and should be more carefully considered. We also argue that the INCUBATE phase should be considered. The exemplar studies show varying levels of success with either commercialization of the solution or distribution of knowledge beyond the research client. However, the success we have had demonstrates what kind of impact DSR projects may have if resources are further invested in establishing the use of the artifact in the client organization or further distributing the use with, e.g., commercialization of the solution. This solutionbased probing further also extends literature on the use of DSRM [3]. This approach builds on the argument made by Peffers et al. [3] that DSR can begin with the existence of a solution, which can launch the research project. Interestingly, Mullarkey and Hevner [11] also proposed a similar extension to the ADR, namely, that ADR can begin with different kinds of entry points for research.

This paper also depicts how to use solution-based probing to advance from developing results that lead to proof-of-concept validation with the INITIATE phase and to proof-of-value validation with the INTERVENE phase, and finally, to proof-of-use validation with the INCUBATE phase. Thus, this work further elaborates Nunamaker et al.'s [1] thoughts on how to achieve rigor and relevance while conducting DSR, and more specifically, using a solution-based probing approach for this. This opens up the debate whether we should develop practices for continuous evaluation in DSR versus the stage-gate approach of the DSRM [3]. Here, we see an opportunity where the ADR literature, including our solution-based probing, may help advance how DSR is evaluated in general [12]. We see here the movement between different types of validation [1] as an intriguing way forward.

Based on these findings, especially with the group support systems-based studies with the U.S. public schools in Washington, D.C., and the USS Coronado, we have found that the work done with the last mile, that is, how to develop proof of use, may bring highly interesting theoretical findings. For example, the USS Coronado launched a research stream that developed collaboration technologies to assist decision making [9] that led to major breakthroughs in this area.

The SmartProtector project also advanced theoretical knowledge in several areas. Most fundamentally, we now have a deeper understanding of the nature of benevolent (i.e., without financial interest) advice-giving. While in the past advicegiving had been conceptualized as knowledge transfer from the advisor to the advisee [13], we could see that progressive advisors actually strive to engage in joint problem solving with the advisees [14]. This was in line with the efforts of the managers responsible for managing the advisees. However, as we built a tool to support joint problem solving, the response from the field quickly showed that the approaches proposed by management and large parts of the literature in the field [15] were too rigid and did not reach far enough. Advice-giving is not just joint problem solving but also has an emotional aspect. Thus, we reconceptualized advice-giving as an act of persuasion [16], that is, striving not only for "enablement" but also for "motivation." In doing so, we discovered a new class of systems and persuasive advisory support, and we extended the discourse on persuasive technologies by introducing the concept of persuasive practices [8]. Most of those insights also apply to other areas of advice-giving, such as financial advisory services. This opens up the opportunity for a comprehensive design theory on advice-giving.

The practical impacts of solution-based probing are also something typically researchers do not see happen. For example, with the USS Coronado we saw how a globally operating organization with several hundreds of thousands of employees started using the developed approach for their daily operations. Another example is how solution-based probing changed how the governance of a major municipality, Stuttgart (a city of about 600,000 inhabitants), is conducted. Equally impressive are the results with the Swiss Police and the Canton of Zürich where commercial software was rolled out recently. Finally, with the U.S. public school study, we were able to significantly improve the learning results of the students and made an even more radical impact on the school dropout rate from $50 \%$ to zero.

\section{Concluding remarks}

Solution-based probing guides researchers toward creating innovations with proof of value and proof of use. This instantiation of action design research facilitates the contribution of design knowledge with high external validity. This is achieved by proposing a theory-based solution that is sharpened and refined in multiple ongoing intervention efforts. The rich observation of human use of technology provides inspiration for new or revised theory, which in turn, informs better design choices.

The four exemplars of solution-based probing research programs demonstrate that pragmatic design research can produce rigorous scientific contributions, and rigorous science can produce novel, generalizable information systems solutions and services. Drawing on extensive theory-based and field-validated design 
knowledge, our research clients were able to charter professional development projects to build operational information system that produced better outcomes with less user resistance. The success of these studies helped us to forge ongoing relationships with the client organizations, which gave us in turn robust venues for conducting rigorous, credible evaluations of the systems, and so to fulfill part of a university's mission to create pragmatic value for society.

Although its benefits are attractive, solution-based probing is complex. It is not a single study, but an ongoing program of research with multiple stakeholders. To clarify its challenges we proposed a phase model to highlight the activities necessary to a solution-based probing approach in the context of action design research. Researchers work to conceive a viable solution, then to find field partners with whom to launch field-based cycles of exploration, design, evaluation, and theorizing to shape a generalizable solution over time. Researchers must pay particular attention to initiating pilot studies designed to produce both scholarly and pragmatic contributions as they work toward developing self-sustaining and growing communities of use around a sustainable solution, and significant contributions to scientific knowledge. All should take place in the context of the build-interveneevaluate cycle, and the reflection/learning activities proposed by Sein et al. [2].

Although we find the detailed phase model reported here to be a valuable guide for conducting high-impact DSR, more work must be done to codify research design patterns and best practices for solution-based probing to produce findings that are both rigorous and relevant. In future research, it may be useful, for instance to analyze the solution-probing research programs of other researchers, with a particular focus on initiating, sustaining, and concluding stakeholder and user engagement in fieldbased settings, for designing studies to both rigorous and relevant, and for gathering, securing, and analyzing data to serve both of those goals, because with a solution-based probing approach, rigor and relevance need not be mutually exclusive.

\section{References}

[1] Nunamaker Jr., J.F., R.O. Briggs, D.C. Derrick, and G. Schwabe, "The Last Research Mile: Achieving Both Rigor and Relevance in Information Systems Research", Journal of Management Information Systems, 2015, pp. 10-47.

[2] Sein, M.K., O. Henfridsson, S. Purao, M. Rossi, and R. Lindgren, "Action Design Research", MIS Quarterly, 2011, pp. 37-56.
[3] Peffers, K., T. Tuunanen, M.A. Rothenberger, and S. Chatterjee, "A Design Science Research Methodology for Information Systems Research", Journal of Management Information Systems, 2007, pp. 45-77.

[4] Schwabe, G. and H. Krcmar, "Piloting Socio-Technical Innovation", in: Proceedings of the 8th European Conference on Information Systems, 27, Wirtschaftsuniversität Wien, Vienna, 2000.

[5] Etzkowitz, H., A. Webster, C. Gebhardt, and B.R.C. Terra, "The Future of the University and the University of the Future: Evolution of Ivory Tower to Entrepreneurial Paradigm", Research Policy, 2000, pp. 313-330.

[6] Witte, E., "Feldexperimente als Innovationstest-die Pilot-projekte zu neuen Medien", Zeitschrift für betriebswirtschaftliche Forschung, 1997, pp. 419-436.

[7] Schwabe, G. and H. Krcmar, "Digital Material in a Political Work Context-The Case of Cuparla", in: Proceedings of the 8th European Conference on Information Systems, 150, Wirtschaftsuniversität Wien, Vienna, 2000.

[8] Schwabe, G. and H. Krcmar, "Wettbewerb als Einfuehrungsstrategie von Telekooperation fuer Entscheidungstraeger - Erfahrungen aus dem Projekt Cuparla", Wirtschaftsinformatik, 1998, pp. 200-204.

[9] Dolata, M., T. Comes, B. Schenk, and G. Schwabe, "Persuasive Practices: Learning from Home Security Advisory Services", in: International Conference on Persuasive Technology, Springer, Cham, 2016, pp. 176-188.

[10] Briggs, R. O., M. Adkins, D. Mittleman, J. Kruse, S. Miller, and J.F. Nunamaker Jr, "A Technology Transition Model Derived from Field Investigation of GSS Use Aboard the USS Coronado", Journal of Management Information Systems, 1998, pp. 151-195.

[11] Mullarkey, M. and A. Hevner, "An Elaborated Action Design Research Process Model", European Journal of Information Systems, 2018, available online.

[12] Venable, J., J. Pries-Heje, and R. Baskerville, "FEDS: A Framework for Evaluation in Design Science Research", European Journal of Information Systems, 2016, pp. 77-89.

[13] Drew, P. and J. Heritage, Talk at Work: Interaction in Institutional Settings, Cambridge University Press, Cambridge, 1992.

[14] Dolata, M. and G. Schwabe, "Tuning in to More Interactivity-Learning from IT Support for Advisory Service Encounters", i-com, De Gruyter, Berlin, 2017, pp. 23-33.

[15] Oehler, A. and D. Kohlert, "Financial Advice Giving and Taking-Where Are the Market's Self-healing Powers and a Functioning Legal Framework When We Need Them?", Journal of Consumer Policy, 2009, pp. 91-116.

[16] Comes, T. and G. Schwabe, "How to Diminish Advice discounting with Mobile Multimedia Interventions", in: Proceedings of the 24th European Conference on Information Systems, Research Paper 40, The association for Information Systems, Istanbul, 2016. 